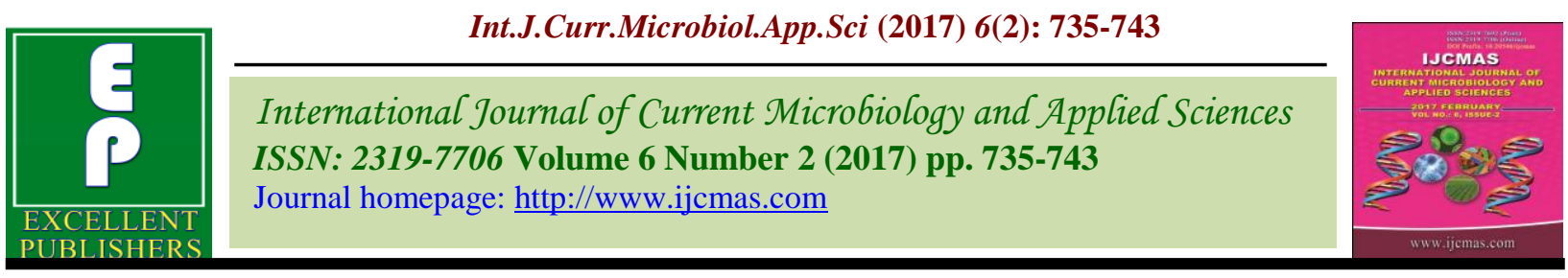

Original Research Article

http://dx.doi.org/10.20546/ijcmas.2017.602.081

\title{
Microbial Response to Different Herbicides Application in Maize for Effective Weed Management
}

\author{
Tapas Ranjan Sahoo $^{{ }^{*}}$, U.K. Hulihalli ${ }^{2}$, R.K. Paikaray ${ }^{1}$ and U. Mohapatra ${ }^{3}$ \\ ${ }^{1}$ Department of Agronomy, CA, OUAT, Bhubaneswar, Odisha, India \\ ${ }^{2}$ Department of Agronomy, CA, UAS, Dharwad, Karnataka, India \\ ${ }^{3}$ Department of Agricultural Economics, CA, OUAT, Bhubaneswar, Odisha, India \\ *Corresponding author
}

A B S T R A C T

Keywords

Herbicides,

Microbial activities,

Grain yield, Weed

control efficiency,

Maize.

Article Info

Accepted:

18 January 2017

Available Online:

10 February 2017
A field experiment was conducted at Main Agricultural Research Station (MARS), Agriculture College, Dharwad to study the "effect of sequential application of herbicides on microbial activities and yield of maize". Six herbicides namely glyphosate, paraquat, glufosinate ammonium, saflufenacil, halosulfuron and imazathapyr were tried as a post emergence in addition to pre-emergence application of atrazine. Totally nine treatments were tested using Randomized Block Design having three replications with three controls (Weedy check, weed free check and standard recommended package of practices (RPP).At all the growth stages weed free check was recorded higher weed control efficiency (WCE) than rest of the weed control methods. However after application of post emergence herbicides atrazine at $1 \mathrm{~kg} \mathrm{ha}^{-1} \mathrm{fb}$ glyphosate at $2.5 \mathrm{~kg} \mathrm{ha}^{-1}$ was recorded with WCE of 92.12 percent next to weed free check. Weed free check was recorded significantly higher grain yield $\left(84.59 \mathrm{q} \mathrm{ha}^{-1}\right)$, stover yield $\left(114.10 \mathrm{q} \mathrm{ha}^{-1}\right)$ and harvest index $(42.57 \%)$. At different growth stages of maize both pre and post emergence application of herbicides, all the enzymatic activities in the soil was reduced as compared to untreated plots.

\section{Introduction}

Management of weeds is considered to be an important factor for achieving higher productivity as weed problem is more severe during continuous rains in early stages of maize growth which cannot be controlled by traditional and cultural practices alone due to too much wetness and labour scarcity. Weed infestation is one of the major constraints for low yield of maize as weeds compete with crop plants for essential inputs. Weed depletes 30-40 per cent of applied nutrients from the soil. The quantities of growth factors used by weeds are thus unavailable to the crop. Sequential use of pre and post emergent herbicides at temporal variation may help in avoiding the problem of weeds throughout the maize growth stages. The increased use of pesticides in agricultural soils causes the contamination of the soil with toxic chemicals. When pesticides are applied, the possibilities exist that these chemicals may exert certain effects on non-target organisms, including soil microorganisms. The microbial biomass plays an important role in the soil ecosystem where they fulfill a crucial role in nutrient cycling and decomposition. During the past four decades, a large number of herbicides have been introduced as pre and 
post-emergent weed killers in many countries of the world. As farmers continue to realize the usefulness of herbicides, larger quantities are applied to the soil. But the fate of these compounds in the soils is becoming increasingly important since they could be leached, in which case groundwater is contaminated or immobile, and persist on the top soil. These herbicides could then accumulate to toxic levels in the soil and become harmful to microorganisms, plant, wild life and man. There is an increasing concern that herbicides not only affect the target organisms (weeds) but also the microbial communities present in soils, and these non-target effects may reduce the performance of important soil functions. The current study was taken up with the objectives to find out efficacy of pre and post emergent herbicides at temporal variation to manage the weeds in maize and to see the effect of herbicides on soil microbes.

\section{Materials and Methods}

A field experiment was conducted during kharif 2014 at Main Agricultural Research Station (MARS), College of Agriculture, Dharwad which is situated at $15^{\circ} 29^{\prime} \mathrm{N}$ latitude, $74^{0} 59^{\prime}$ ' $\mathrm{E}$ longitudes and at an altitude of $689 \mathrm{~m}$ above mean sea level and it comes under Northern Transition Zone (Zone-8) of Karnataka. The soil of the experimental site was black clayey soil with $\mathrm{pH}$ 7.1, available $\mathrm{N}, \mathrm{P}_{2} \mathrm{O}_{5}$ and $\mathrm{K}_{2} \mathrm{O} 229,34$, and $484 \mathrm{~kg} / \mathrm{ha}$, respectively. Treatments comprised of six herbicides namely Glyphosate $(2.5 \mathrm{~kg} / \mathrm{ha})$, Paraquat (1 kg/ha), Glufosinate ammonium (0.375 kg/ha), Saflufenacil (75 g/ha), Halosulfuron (90 g/ha) and Imazathapyr (75 $\mathrm{g} / \mathrm{ha}$ ) were tried as post emergence (Directed spray) in addition to pre-emergence application of Atrazine $(1 \mathrm{~kg} / \mathrm{ha})$ along with Atrazine+ 2 interculture +1 hand weeding, weed free and weedy check. Totally nine treatments were tested using Randomized Block Design having three replications. The test variety was $900 \mathrm{M}$ Gold and the crop received a rainfall of $633.5 \mathrm{~mm}$ during crop growing period. The weed infestation was predominantly consisted of grassy weeds, broad leaved weeds and sedges. The dehydrogenase activity, phosphatase activity and urease activity in the soil samples was determined by following the procedure as described by Casida et al. (1964), Evazi and Tabatabai (1979) and Pancholy and Rice (1973), respectively.

\section{Weed control efficiency $(\%)$}

Weed control efficiency (WCE) denotes the magnitude of weed reduction due to the weed control treatments. The weed control efficiency was calculated by using the formula given by Misra and Tosh (1979).

Dry weight of weeds in

Dry weight of weeds weedy check $(\mathrm{g}) \quad$ in treatment plot $(\mathrm{g})$

WCE $(\%)=$

Dry weight of weeds in weedy check $(g)$

\section{Grain yield $\left(\mathbf{q}\right.$ ha $\left.^{-1}\right)$}

The cobs from the net plot were harvested at physiological maturity and they were dehusked. The dehusked cobs were air-dried and grains were separated from the cob by shelling and weighed the shelled grains after complete drying and taken as grain yield per plot. The grain yield was expressed in $\mathrm{q} \mathrm{ha}^{-1}$.

\section{Stover yield (q $\left.\mathbf{h a}^{-1}\right)$}

Stover yield was recorded after complete sun drying of stalks from each net plot and expressed in $\mathrm{q} \mathrm{ha}^{-1}$. 


\section{Harvest index (\%)}

Harvest index was defined as the ratio of economic yield to biological yield and expressed in per cent. Harvest efficiency was estimated as per the formula suggested by Donald (1962).

$$
\mathrm{HI}(\%)=\frac{\text { Economic yield }\left(\mathrm{q} \mathrm{ha}^{-1}\right)}{\text { Biological yield }\left(\mathrm{q} \mathrm{ha}^{-1}\right)}
$$

\section{Results and Discussion}

\section{Weed Control Efficiency}

Weed control efficiency (WCE) differed significantly with different weed control methods at various crop growth stages (Table $1)$.

In weed control efficiency (WCE), total weed dry weight was taken into account which consists of different weed species which will be varying in their proportions. As such it will not reveal the individual weed species effect. WCE of treatment normally decreases over time or as the date of observation advances. Performance of crop is directly proportional to the weed control efficiency and inversely proportional to the weed index. In the present study, at 20, 40 and 60 DAS and also at harvest, $\mathrm{T}_{8}$ (weed free check) was recorded higher WCE than rest of the weed control methods. There was no significant difference among herbicide treatments before application of post emergence herbicides. After pre emergence application of Atrazine, the weed control efficiency in each herbicides treatment did not varied significantly as common herbicide was used in these treatments. But at 60 DAS, $\mathrm{T}_{8}$ (weed free check) was recorded with higher WCE $(97.26 \%)$ as compared to other treatments. The next best treatments were $\mathrm{T}_{1}$ (Atrazine at $1 \mathrm{~kg} \mathrm{ha}^{-1}$ fb Glyphosate at $2.5 \mathrm{~kg} \mathrm{ha}^{-1}$ ) (92.12\%), $\mathrm{T}_{3}$ (Atrazine at $1 \mathrm{~kg} \mathrm{ha}^{-1} \mathrm{fb}$
Glufosinate ammonium at $0.375 \mathrm{~kg} \mathrm{ha}^{-1}$ ) $(91.73 \%), \mathrm{T}_{4}$ (Atrazine at $1 \mathrm{~kg} \mathrm{ha}^{-1} \mathrm{fb}$ Saflufenacil at $\left.75 \mathrm{~g} \mathrm{ha}^{-1}\right)(90.04 \%)$ and $\mathrm{T}_{2}$ (Atrazine at $1 \mathrm{~kg} \mathrm{ha}^{-1} \mathrm{fb}$ Paraquat at $1 \mathrm{~kg} \mathrm{ha}^{-1}$ ) (89.14\%) which were found on par with each other. It was due to lower weed population and total dry weight of weeds in these treatments due to better control of weeds following exposure to post emergent treatment. Similar kind of results was observed by Shantveerayya et al., (2012) and Hatti et al., (2014).

\section{Yield and yield components}

Yield is the net result of various interactions i.e. soil characters, weather parameters, crop weed competition and various metabolic and biochemical interactions taking place throughout the plant growth. Maize grain yield is also influenced by dry matter accumulation in different parts especially in reproductive part and yield components.

Yield of maize varied significantly among various weed control treatments (Table 1). Significantly higher grain yield $\left(84.59 \mathrm{q} \mathrm{ha}^{-1}\right)$ was recorded in $\mathrm{T}_{8}$ (weed free check) which was 87.68 per cent higher than weedy check $\left(45.07 \mathrm{q} \mathrm{ha}^{-1}\right.$ ) followed by $\mathrm{T}_{4}$ (Atrazine at 1 $\mathrm{kg} \mathrm{ha}^{-1} \mathrm{fb}$ Saflufenacil at $\left.75 \mathrm{~g} \mathrm{ha}^{-1}\right)(81.38 \mathrm{q}$ $\mathrm{ha}^{-1}$ ) which was 80.56 per cent higher than the weedy check. The next best treatment was $T_{2}$ (Atrazine at $1 \mathrm{~kg} \mathrm{ha}^{-1} \mathrm{fb}$ Paraquat at $1 \mathrm{~kg} \mathrm{ha}^{-1}$ ) which was recorded with grain yield of 70.56 $\mathrm{q} \mathrm{ha}{ }^{-1}$ (56.55 per cent more than weedy check). Significantly higher yield was due to minimum crop-weed competition throughout the crop growth period, thus enabling the crop for maximum utilization of nutrients, moisture, light and space which had influence on growth components and yield components $v i z$, cob length, cob girth, test weight, grain weight plant $^{-1}$, shelling per cent and harvest index as less weed competition and lesser phytotoxicity injury as well as better growth 
characteristics (Table 2). The lowest grain yield (45.07 $\mathrm{q} \mathrm{ha}^{-1}$ ) was recorded with weedy check $\left(\mathrm{T}_{9}\right)$ as a consequence of highest removal of nutrients and moisture by weeds and severe crop weed competition resulting in poor source-sink relationship with poor yield components. Similar types of results were reported by Chougala (2013), Singh et al. (2014) and Patil (2014).

Among the herbicide treatments significantly higher grain yield was recorded in $\mathrm{T}_{4}$ (Atrazine at $1 \mathrm{~kg} \mathrm{ha}^{-1} \mathrm{fb}$ Saflufenacil at $75 \mathrm{~g}$ $\left.\mathrm{ha}^{-1}\right)\left(81.38 \mathrm{q} \mathrm{ha}^{-1}\right)$ which was 80.56 per cent higher than the weedy check. The next best treatment was $\mathrm{T}_{2}$ (Atrazine at $1 \mathrm{~kg} \mathrm{ha}^{-1} \mathrm{fb}$ Paraquat at $1 \mathrm{~kg} \mathrm{ha}^{-1}$ ) which was recorded with grain yield of $70.56 \mathrm{q} \mathrm{ha}^{-1}$ (56.55 per cent more than weedy check) this was due to improved growth and yield attributes. Thus, the improvement in growth and yield components was as a consequence of lower crop-weed competition, which shifted the balance in favour of crop in the utilization of nutrients, moisture, light and space. These results are in conformity with the findings of Saini and Angiras (1998), Sreenivas and Satyanarayana (1994) and Kamble et al. (2005).

Significant differences in stover yield $\left(\mathrm{q} \mathrm{ha}^{-1}\right)$ were recorded with respect to different weed control treatments (Table 3). $\mathrm{T}_{8}$ (weed free check) was recorded significantly higher stover yield (114.10 $\left.\mathrm{q} \mathrm{ha}^{-1}\right)$ which was 71.83 per cent higher over weedy check (66.40 q ha ${ }^{1}$ ) and it was on par with $\mathrm{T}_{4}$ (Atrazine at $1 \mathrm{~kg}$ $\mathrm{ha}^{-1} \mathrm{fb}$ Saflufenacil at $75 \mathrm{~g} \mathrm{ha}^{-1}$ ) having stover yield $109.80 \mathrm{q} \mathrm{ha}^{-1}$ (65.36 per cent higher over weedy check). The next best treatment was $\mathrm{T}_{2}$ (Atrazine at $1 \mathrm{~kg} \mathrm{ha}^{-1} \mathrm{fb}$ Paraquat at 1 $\mathrm{kg} \mathrm{ha}^{-1}$ ) which was recorded stover yield of $102.10 \mathrm{q} \mathrm{ha}^{-1}$ (53.76 per cent higher over weedy check). Higher stover yield may be attributed to higher dry matter production and its accumulation in leaves, stem, reproductive parts and also other growth attributes such as plant height, number of leaves, leaf area, leaf area index. These results are in conformity with the findings of Ishrat et al. (2012) and Chougala (2013).

\section{Effect of herbicides on microbial activities}

Dehydrogenase activity ( $\mu \mathrm{g}$ TPF $\mathrm{g}^{-1}$ soil
day $^{-1}$ )

At 7 DAT, the non-treated control plots i.e. $\mathrm{T}_{8}$ (weed free check) and $\mathrm{T}_{9}$ (weedy check) were recorded significantly higher dehydrogenase activity (34.66 and $34.30 \mu \mathrm{g} \mathrm{TPF} \mathrm{g}^{-1}$ soil day ${ }^{-1}$ respectively) as compared to other weed control methods. Among the herbicide treatments there were no significant differences with respect to dehydrogenase activity but all treatments showed significantly lower dehydrogenase activity compared to control. Numerically higher dehydrogenase activity $\left(25.59 \mu \mathrm{g}\right.$ TPF g ${ }^{-1}$ soil day $^{-1}$ ) was registered in $T_{4}$ (Atrazine at $1 \mathrm{~kg}$ $\mathrm{ha}^{-1} \mathrm{fb}$ Saflufenacil at $75 \mathrm{~g} \mathrm{ha}^{-1}$ ).

At $15 \mathrm{DAT}, \mathrm{T}_{4}$ (Atrazine at $1 \mathrm{~kg} \mathrm{ha}^{-1} \mathrm{fb}$ Saflufenacil at $75 \mathrm{~g} \mathrm{ha}^{-1}$ ) was recorded significantly higher dehydrogenase activity $\left(38.20 \mu \mathrm{g} \mathrm{TPF} \mathrm{g}^{-1}\right.$ soil day $\left.{ }^{-1}\right)$ which was on par with $\mathrm{T}_{2}$ (Atrazine at $1 \mathrm{~kg} \mathrm{ha}^{-1} \mathrm{fb}$ Paraquat at $\left.1 \mathrm{~kg} \mathrm{ha}^{-1}\right)\left(36.22 \mu \mathrm{g}\right.$ TPF g ${ }^{-1}$ soil day $\left.{ }^{-1}\right)$ andT $\mathrm{T}_{5}$ (Atrazine at $1 \mathrm{~kg} \mathrm{ha}^{-1} \mathrm{fb}$ Halosulfuron at $\left.90 \mathrm{~g} \mathrm{ha}^{-1}\right)$ (35.48 $\mu \mathrm{g}$ TPF $\mathrm{g}^{-1}$ soil day ${ }^{-1}$ ). Significantly lower dehydrogenase activity (29.25 $\mu \mathrm{g} \mathrm{TPF} \mathrm{g}^{-1}$ soil day $\left.{ }^{-1}\right)$ was recorded in $\mathrm{T}_{9}$ (weedy check) which was on par with $\mathrm{T}_{6}$ (Atrazine at $1 \mathrm{~kg} \mathrm{ha}^{-1} \mathrm{fb}$ Imazathapyr at $75 \mathrm{~g}$ $\left.\mathrm{ha}^{-1}\right)\left(31.74 \mu \mathrm{g} \mathrm{TPF} \mathrm{g}^{-1}\right.$ soil day $\left.^{-1}\right)$.

\section{Post emergence application}

At 7 DAT, $\mathrm{T}_{8}$ (weed free check) was recorded significantly higher dehydrogenase activity $\left(39.55 \mu \mathrm{g} \mathrm{TPF} \mathrm{g}^{-1}\right.$ soil day $\left.{ }^{-1}\right)$ followed by $\mathrm{T}_{9}$ (weedy check) and $\mathrm{T}_{7}$ (Atrazine at $1 \mathrm{~kg} \mathrm{ha}^{-1}+$ 
$2 \mathrm{IC}+1 \mathrm{HW})\left(35.06\right.$ and $34.13 \mu \mathrm{g}$ TPF $\mathrm{g}^{-1}$ soil day ${ }^{-1}$ respectively). The herbicides treated treatments were recorded significantly lower dehydrogenase activity compared to control plots. $\mathrm{T}_{3}$ (Atrazine at $1 \mathrm{~kg} \mathrm{ha}^{-1} \mathrm{fb}$ Glufosinate ammonium at $0.375 \mathrm{~kg} \mathrm{ha}^{-1}$ ) recorded lower dehydrogenase activity $\left(22.30 \mu \mathrm{g} \mathrm{TPF} \mathrm{g}^{-1}\right.$ soil day $^{-1}$ ) which was on par with $\mathrm{T}_{4}$ (Atrazine at 1 $\mathrm{kg} \mathrm{ha}^{-1} \mathrm{fb}$ Saflufenacil at $\left.75 \mathrm{~g} \mathrm{ha}^{-1}\right)$.

At 15 DAT, $\mathrm{T}_{8}$ (weed free check) was recorded significantly higher dehydrogenase activity (41.83 $\mu \mathrm{g}$ TPF g ${ }^{-1}$ soil day ${ }^{-1}$ ) which was on par with $\mathrm{T}_{2}$ (Atrazine at $1 \mathrm{~kg} \mathrm{ha}^{-1} \mathrm{fb}$ Paraquat at $\left.1 \mathrm{~kg} \mathrm{ha}^{-1}\right)\left(39.47 \mu \mathrm{g}\right.$ TPF g ${ }^{-1}$ soil day $^{-1}$ ). Whereas, $\mathrm{T}_{6}$ (Atrazine at $1 \mathrm{~kg} \mathrm{ha}^{-1} \mathrm{fb}$ Imazathapyr at $75 \mathrm{~g} \mathrm{ha}^{-1}$ ) was recorded significantly lower dehydrogenase activity (31.74 $\mu \mathrm{g}$ TPF $\mathrm{g}^{-1}$ soil day $\left.^{-1}\right)$ which was on par with $\mathrm{T}_{7}$ (Atrazine at $1 \mathrm{~kg} \mathrm{ha}^{-1}+2 \mathrm{IC}+1$ $\mathrm{HW}$ ) and $\mathrm{T}_{4}$ (Atrazine at $1 \mathrm{~kg} \mathrm{ha}^{-1} \mathrm{fb}$ Saflufenacil $\left.75 \mathrm{~g} \mathrm{ha}^{-1}\right)$.

\section{Phosphatase activity ( $\mu$ g pnp $\mathrm{g}^{-1}$ soil $\mathrm{hr}^{-1}$ )}

\section{Pre emergence application}

At 7 DAT, the non-treated control plots i.e., $\mathrm{T}_{8}$ (weed free check) and $\mathrm{T}_{9}$ (weedy check) were recorded significantly higher phosphatase activity $\left(90.60 \mu \mathrm{g}_{\mathrm{pnp} \mathrm{g}} \mathrm{g}^{-1}\right.$ soil hr ${ }^{1}$ each) as compared to other weed control methods. $\mathrm{T}_{3}$ (Atrazine at $1 \mathrm{~kg} \mathrm{ha}^{-1} \mathrm{fb}$ Glufosinate ammonium at $0.375 \mathrm{~kg} \mathrm{ha}^{-1}$ ) was recorded significantly lower phosphatase activity (46.67 $\mu \mathrm{g}$ pnp $\mathrm{g}^{-1}$ soil $\mathrm{hr}^{-1}$ ). Among rest of the treatments there were no significant differences with respect to phosphatase activity but all treatments showed significantly lower phosphatase activity compared to control.

At 15 DAT, $\mathrm{T}_{8}$ (weed free check) was recorded significantly higher phosphatase activity (106.43 $\mu \mathrm{g}$ pnp g ${ }^{-1}$ soil $\mathrm{hr}^{-1}$ ) which was on par with $\mathrm{T}_{9}$ (weedy check) $(100.27 \mu \mathrm{g}$ pnp $\mathrm{g}^{-1}$ soil $\mathrm{hr}^{-1}$ ). Whereas, $\mathrm{T}_{5}$ (Atrazine at 1 $\mathrm{kg} \mathrm{ha}^{-1} \mathrm{fb}$ Halosulfuron at $90 \mathrm{~g} \mathrm{ha}^{-1}$ ) was recorded significantly lower phosphatase

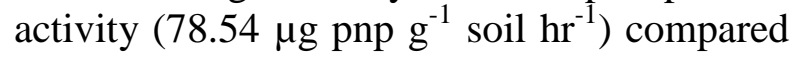
to control. Among rest of the treatments there were no significant differences with each other with respect to phosphatase activity but numerically higher phosphatase activity was recorded in $\mathrm{T}_{1}$ (Atrazine at $1 \mathrm{~kg} \mathrm{ha}^{-1} \mathrm{fb}$ Glyphosate at $\left.2.5 \mathrm{~kg} \mathrm{ha}^{-1}\right)(92.23 \mu \mathrm{g} \mathrm{pnp} \mathrm{g}$ soil $\mathrm{hr}^{-1}$ ).

\section{Post emergence application}

At 7 DAT, T $_{8}$ (weed free check) was recorded significantly higher phosphatase activity $\left(118.51 \mu \mathrm{g}\right.$ pnp $\mathrm{g}^{-1}$ soil $\left.\mathrm{hr}^{-1}\right)$ as compared to other weed control methods. But, $\mathrm{T}_{6}$ (Atrazine at $1 \mathrm{~kg} \mathrm{ha}^{-1} \mathrm{fb}$ Imazathapyr at $75 \mathrm{~g} \mathrm{ha}^{-1}$ ) was recorded significantly lower phosphatase activity (87.41 $\mu \mathrm{g} \mathrm{pnp} \mathrm{g}^{-1}$ soil $\mathrm{hr}^{-1}$ ) which was on par with all other herbicides treatments except $\mathrm{T}_{3}$ (Atrazine at $1 \mathrm{~kg} \mathrm{ha}^{-1}$ fb Glufosinate ammonium at $0.375 \mathrm{~kg} \mathrm{ha}^{-1}$ ).

At 15 DAT, $\mathrm{T}_{2}$ (Atrazine at $1 \mathrm{~kg} \mathrm{ha}^{-1} \mathrm{fb}$ Paraquat at $1 \mathrm{~kg} \mathrm{ha}^{-1}$ ) was recorded significantly higher phosphatase activity (119.40 $\mu \mathrm{g}_{\mathrm{pnp} \mathrm{g}} \mathrm{g}^{-1}$ soil $\mathrm{hr}^{-1}$ ) which was on par with $\mathrm{T}_{1}$ (Atrazine at $1 \mathrm{~kg} \mathrm{ha}^{-1} \mathrm{fb}$ Glyphosate at $\left.2.5 \mathrm{~kg} \mathrm{ha}^{-1}\right)\left(117.65 \mu \mathrm{g}\right.$ pnp g ${ }^{-1}$ soil hr$\left.{ }^{-1}\right)$ and $\mathrm{T}_{8}$ (weed free check) (114.14 $\mu \mathrm{g} \mathrm{pnp} \mathrm{g}^{-1}$ soil $\mathrm{hr}^{-1}$ ). Whereas, $\mathrm{T}_{9}$ (weedy check) was recorded significantly lower phosphatase activity (104.46 $\mu \mathrm{g}$ pnp g $\mathrm{g}^{-1}$ soil $\mathrm{hr}^{-1}$ ) which was on par with $T_{3}, T_{5}$ and $T_{6}$.

\section{Ureases activity ( $\mu \mathrm{g} \mathrm{NH} \mathrm{N}^{-1}$ soil day ${ }^{-1}$ )}

\section{Pre emergence application}

At 7 DAT. T $_{9}$ (weedy check) recorded significantly higher urease activity $(3.62 \mu \mathrm{g}$ $\mathrm{NH}_{4} \mathrm{~N} \mathrm{~g}^{-1}$ soil day ${ }^{-1}$ ) which was on par with $\mathrm{T}_{8}$ (weed free check) (3.59 $\mu \mathrm{g} \mathrm{NH}_{4} \mathrm{~N} \mathrm{~g}^{-1}$ soil $\left.\operatorname{day}^{-1}\right)$. 
Table.1 Weed control efficiency as influenced by weed control methods in maize

\begin{tabular}{|c|c|c|c|c|}
\hline \multirow[t]{2}{*}{ Treatments } & \multicolumn{4}{|c|}{ Weed control efficiency $(\%)$} \\
\hline & 20 DAS & 40 DAS & 60 DAS & Harvest \\
\hline $\mathrm{T}_{1}$ - Atrazine at $1 \mathrm{~kg} \mathrm{ha}^{-1} \mathrm{fb}$ Glyphosate at $2.5 \mathrm{~kg} \mathrm{ha}^{-1}$ (Directed spray) & 72.40 & 43.71 & 92.12 & 85.56 \\
\hline $\mathrm{T}_{2}$ - Atrazine at $1 \mathrm{~kg} \mathrm{ha}^{-1} \mathrm{fb}$ Paraquat at $1 \mathrm{~kg} \mathrm{ha}^{-1}$ (Directed spray) & 71.27 & 43.68 & 89.14 & 71.89 \\
\hline $\begin{array}{l}\mathrm{T}_{3} \text { - Atrazine at } 1 \mathrm{~kg} \mathrm{ha}^{-1} \mathrm{fb} \text { Glufosinate ammonium at } 0.375 \mathrm{~kg} \mathrm{ha}^{-1} \text { (Directed } \\
\text { spray) }\end{array}$ & 77.36 & 48.18 & 91.73 & 84.84 \\
\hline $\mathrm{T}_{4}$ - Atrazine at $1 \mathrm{~kg} \mathrm{ha}^{-1} \mathrm{fb}$ Saflufenacil at $75 \mathrm{~g} \mathrm{ha}^{-1}$ (Directed spray) & 75.19 & 50.57 & 90.40 & 77.13 \\
\hline $\mathrm{T}_{5}$ - Atrazine at $1 \mathrm{~kg} \mathrm{ha}^{-1} \mathrm{fb}$ Halosulfuron at $90 \mathrm{~g} \mathrm{ha}^{-1}$ (Directed spray) & 73.91 & 42.61 & 81.09 & 69.98 \\
\hline $\mathrm{T}_{6}$ - Atrazine at $1 \mathrm{~kg} \mathrm{ha}^{-1} \mathrm{fb}$ Imazathapyr at $75 \mathrm{~g} \mathrm{ha}^{-1}$ (Directed spray) & 70.37 & 42.75 & 75.56 & 70.43 \\
\hline $\mathrm{T}_{7}$ - Atrazine at $1 \mathrm{~kg} \mathrm{ha}^{-1}+2 \mathrm{IC}+1 \mathrm{HW}(\mathrm{RPP}$ standard check) & 76.81 & 81.50 & 86.62 & 56.46 \\
\hline $\mathrm{T}_{8}-$ Weed free check & 91.89 & 90.19 & 97.26 & 91.55 \\
\hline $\mathrm{T}_{9}-$ Weedy check & - & - & - & - \\
\hline S. Em \pm & 4.96 & 2.73 & 1.54 & 2.42 \\
\hline $\mathrm{CD}(0.05)$ & 14.87 & 8.17 & 4.63 & 7.27 \\
\hline
\end{tabular}

IC: Inter cultivation $\mathbf{H W}$ : Hand Weeding fb: followed by

Note: All the above mentioned post-emergent $\left(T_{1}-T_{6}\right)$ herbicides sprayed as directed spray at 45 DAS

Table.2 Test weight, shelling percentage, grain yield, stover yield and harvest index as influenced by weed control methods in maize

\begin{tabular}{|c|c|c|c|c|c|}
\hline Treatments & $\begin{array}{c}\text { Test } \\
\text { weight } \\
\text { (g) }\end{array}$ & $\begin{array}{c}\text { Shelling } \\
\text { percentage }\end{array}$ & $\begin{array}{c}\text { Grain } \\
\text { yield } \\
\left(\mathbf{q ~ h a} \mathbf{~ h a}^{-1}\right)\end{array}$ & $\begin{array}{c}\text { Stover } \\
\text { yield (q } \\
\left.\text { ha }^{-1}\right)\end{array}$ & $\begin{array}{c}\text { Harvest } \\
\text { index }(\%)\end{array}$ \\
\hline $\mathrm{T}_{1}$ - Atrazine at $1 \mathrm{~kg} \mathrm{ha}^{-1} \mathrm{fb}$ Glyphosate at $2.5 \mathrm{~kg} \mathrm{ha}^{-1}$ (Directed spray) & 33.25 & 70.67 & 59.11 & 83.00 & 41.63 \\
\hline $\mathrm{T}_{2}-$ Atrazine at $1 \mathrm{~kg} \mathrm{ha}^{-1} \mathrm{fb}$ Paraquat at $1 \mathrm{~kg} \mathrm{ha}^{-1}$ (Directed spray) & 33.86 & 70.61 & 70.56 & 102.10 & 40.86 \\
\hline $\mathrm{T}_{3}$ - Atrazine at $1 \mathrm{~kg} \mathrm{ha}^{-1} \mathrm{fb}$ Glufosinate ammonium at $0.375 \mathrm{~kg} \mathrm{ha}^{-1}$ (Directed spray) & 32.90 & 70.61 & 65.83 & 95.30 & 40.87 \\
\hline $\mathrm{T}_{4}$ - Atrazine at $1 \mathrm{~kg} \mathrm{ha}^{-1} \mathrm{fb}$ Saflufenacil at $75 \mathrm{~g} \mathrm{ha}^{-1}$ (Directed spray) & 35.25 & 72.81 & 81.38 & 109.80 & 42.56 \\
\hline $\mathrm{T}_{5}$ - Atrazine at $1 \mathrm{~kg} \mathrm{ha}^{-1} \mathrm{fb}$ Halosulfuron at $90 \mathrm{~g} \mathrm{ha}^{-1}$ (Directed spray) & 33.13 & 70.41 & 67.83 & 98.57 & 40.78 \\
\hline $\mathrm{T}_{6}$ - Atrazine at $1 \mathrm{~kg} \mathrm{ha}^{-1} \mathrm{fb}$ Imazathapyr at $75 \mathrm{~g} \mathrm{ha}^{-1}$ (Directed spray) & 33.21 & 70.64 & 62.67 & 91.30 & 40.70 \\
\hline $\mathrm{T}_{7}-$ Atrazine at $1 \mathrm{~kg} \mathrm{ha}^{-1}+2 \mathrm{IC}+1 \mathrm{HW}$ (RPP standard check) & 33.83 & 71.31 & 68.17 & 94.67 & 41.86 \\
\hline $\mathrm{T}_{8}-$ Weed free check & 35.71 & 73.17 & 84.59 & 114.10 & 42.57 \\
\hline $\mathrm{T}_{9}-$ Weedy check & 31.36 & 69.36 & 45.07 & 66.40 & 40.45 \\
\hline S. Em \pm & 0.39 & 0.29 & 0.68 & 1.63 & 0.34 \\
\hline CD $(0.05)$ & 1.15 & 0.87 & 2.05 & 4.87 & 1.02 \\
\hline
\end{tabular}

IC: Inter cultivation $\mathbf{H W}$ : Hand Weeding fb: followed by

Note: All the above mentioned post-emergent $\left(\mathrm{T}_{1}-\mathrm{T}_{6}\right)$ herbicides sprayed as directed spray at 45 DAS 
Table.3 Dehydrogenase, phosphatase and urease activity as influenced by weed control methods in maize

\begin{tabular}{|c|c|c|c|c|c|c|c|c|c|c|c|c|}
\hline \multirow{3}{*}{ Treatments } & \multicolumn{4}{|c|}{$\begin{array}{l}\text { Dehydrogenase activity } \\
\left(\mu \mathrm{g}^{\mathrm{T} P F} \mathrm{~g}^{-1} \text { soil day }^{-1}\right)\end{array}$} & \multicolumn{4}{|c|}{$\begin{array}{c}\text { Phosphatase activity ( } \mu \mathrm{g} \text { pnp } \\
\mathrm{g}^{-1} \text { soil } \mathrm{hr}^{-1} \text { ) }\end{array}$} & \multicolumn{4}{|c|}{$\begin{array}{c}\text { Urease activity }\left(\mu \mathrm{g} \mathrm{NH}_{4} \mathrm{~N}\right. \\
\left.\mathrm{g}^{-1} \text { soil day }^{-1}\right)\end{array}$} \\
\hline & \multicolumn{2}{|c|}{$\begin{array}{c}\text { Pre } \\
\text { emergence }\end{array}$} & \multicolumn{2}{|c|}{$\begin{array}{c}\text { Post } \\
\text { emergence }\end{array}$} & \multicolumn{2}{|c|}{$\begin{array}{c}\text { Pre } \\
\text { emergence }\end{array}$} & \multicolumn{2}{|c|}{$\begin{array}{c}\text { Post } \\
\text { emergence }\end{array}$} & \multicolumn{2}{|c|}{$\begin{array}{c}\text { Pre } \\
\text { emergence }\end{array}$} & \multicolumn{2}{|c|}{$\begin{array}{c}\text { Post } \\
\text { emergence }\end{array}$} \\
\hline & $\begin{array}{c}7 \\
\text { DAT }\end{array}$ & $\begin{array}{c}15 \\
\text { DAT }\end{array}$ & $\begin{array}{c}7 \\
\text { DAT }\end{array}$ & $\begin{array}{c}15 \\
\text { DAT }\end{array}$ & $\begin{array}{c}7 \\
\text { DAT }\end{array}$ & $\begin{array}{c}15 \\
\text { DAT }\end{array}$ & $\begin{array}{c}7 \\
\text { DAT }\end{array}$ & $\begin{array}{c}15 \\
\text { DAT }\end{array}$ & $\begin{array}{c}7 \\
\text { DAT }\end{array}$ & $\begin{array}{c}15 \\
\text { DAT }\end{array}$ & $\begin{array}{c}7 \\
\text { DAT }\end{array}$ & $\begin{array}{c}15 \\
\text { DAT }\end{array}$ \\
\hline $\mathrm{T}_{1}$ - Atrazine at $1 \mathrm{~kg} \mathrm{ha}^{-1} \mathrm{fb}$ Glyphosate at $2.5 \mathrm{~kg} \mathrm{ha}^{-1}$ (Directed spray) & 24.71 & 33.38 & 26.44 & 35.85 & 55.51 & 92.23 & 95.98 & 117.65 & 2.74 & 3.85 & 3.09 & 3.69 \\
\hline $\mathrm{T}_{2}$ - Atrazine at $1 \mathrm{~kg} \mathrm{ha}^{-1} \mathrm{fb}$ Paraquat at $1 \mathrm{~kg} \mathrm{ha}^{-1}$ (Directed spray) & 24.43 & 36.22 & 26.92 & 39.47 & 56.25 & 85.74 & 93.87 & 119.40 & 2.72 & 4.00 & 2.85 & 3.87 \\
\hline $\mathrm{T}_{3}$ - Atrazine at $1 \mathrm{~kg} \mathrm{ha}^{-1} \mathrm{fb}$ Glufosinate ammonium at $0.375 \mathrm{~kg} \mathrm{ha}^{-1}(\mathrm{DS})$ & 25.30 & 34.61 & 22.30 & 34.95 & 46.67 & 88.99 & 101.13 & 107.89 & 2.62 & 3.77 & 2.79 & 4.03 \\
\hline $\mathrm{T}_{4}-$ Atrazine at $1 \mathrm{~kg} \mathrm{ha}^{-1} \mathrm{fb}$ Saflufenacil at $75 \mathrm{~g} \mathrm{ha}^{-1}$ (Directed spray) & 25.59 & 38.20 & 23.87 & 31.42 & 63.84 & 88.96 & 92.23 & 110.74 & 2.51 & 3.74 & 2.99 & 4.13 \\
\hline $\mathrm{T}_{5}$ - Atrazine at $1 \mathrm{~kg} \mathrm{ha}^{-1} \mathrm{fb}$ Halosulfuron at $90 \mathrm{~g} \mathrm{ha}^{-1}$ (Directed spray) & 25.00 & 35.48 & 26.18 & 34.42 & 55.51 & 78.54 & 88.15 & 110.68 & 2.39 & 3.69 & 3.29 & 4.63 \\
\hline $\mathrm{T}_{6}$ - Atrazine at $1 \mathrm{~kg} \mathrm{ha}^{-1} \mathrm{fb}$ Imazathapyr at $75 \mathrm{~g} \mathrm{ha}^{-1}$ (Directed spray) & 24.28 & 31.74 & 24.86 & 30.45 & 56.28 & 83.33 & 87.41 & 108.99 & 2.51 & 3.61 & 2.73 & 3.72 \\
\hline $\mathrm{T}_{7}$ - Atrazine at $1 \mathrm{~kg} \mathrm{ha}^{-1}+2 \mathrm{IC}+1 \mathrm{HW}$ (RPP standard check) & 24.72 & 33.92 & 34.13 & 32.59 & 60.77 & 82.50 & 110.68 & 106.43 & 2.39 & 3.88 & 4.29 & 4.16 \\
\hline $\mathrm{T}_{8}-$ Weed free check & 34.66 & 34.13 & 39.55 & 41.83 & 90.60 & 106.43 & 118.51 & 114.14 & 3.59 & 4.05 & 4.74 & 4.62 \\
\hline $\mathrm{T}_{9}-$ Weedy check & 34.30 & 29.25 & 35.06 & 34.95 & 90.60 & 100.27 & 103.99 & 104.46 & 3.62 & 3.90 & 4.29 & 4.21 \\
\hline $\mathbf{S . ~ E m} \pm$ & 0.54 & 1.11 & 0.98 & 0.88 & 2.90 & 3.04 & 3.74 & 1.83 & 0.10 & 0.11 & 0.07 & 0.11 \\
\hline CD (0.05) & 1.62 & 3.32 & 2.93 & 2.65 & 8.69 & 9.12 & 11.21 & 5.49 & 0.29 & 0.34 & 0.20 & 0.32 \\
\hline
\end{tabular}

IC: Inter cultivation HW: Hand Weeding fb: followed by DAT: Days after herbicide treatment DS: Directed spray

Note: All the above mentioned post-emergent $\left(\mathrm{T}_{1}-\mathrm{T}_{6}\right)$ herbicides sprayed as directed spray at 45 DAS 
Among the herbicide treatments $\mathrm{T}_{1}$ (Atrazine at $1 \mathrm{~kg} \mathrm{ha}^{-1} \mathrm{fb}$ Glyphosate at $2.5 \mathrm{~kg} \mathrm{ha}^{-1}$ ) recorded significantly higher urease activity (2.74 $\mu \mathrm{g} \mathrm{NH}_{4} \mathrm{~N} \mathrm{~g}^{-1}$ soil day ${ }^{-1}$ ) which was on par with all other treatments except $\mathrm{T}_{5}$ (Atrazine at $1 \mathrm{~kg} \mathrm{ha}^{-1} \mathrm{fb}^{-}$Halosulfuron at $90 \mathrm{~g}$

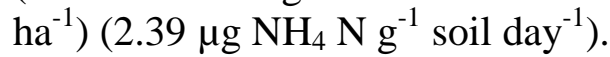

At 15 DAT, there were no significant differences among treatments with respect to urease activity except $\mathrm{T}_{6}$ (Atrazine at $1 \mathrm{~kg} \mathrm{ha}^{-1}$ $\mathrm{fb}$ Imazathapyr at $75 \mathrm{~g} \mathrm{ha}^{-1}$ ) and $\mathrm{T}_{5}$ (Atrazine at $1 \mathrm{~kg} \mathrm{ha}^{-1} \mathrm{fb}$ Halosulfuron at $90 \mathrm{~g} \mathrm{ha}^{-1}$ ) which were recorded significantly lower urease activity (3.61 and $3.69 \mu \mathrm{g} \mathrm{NH}_{4} \mathrm{~N} \mathrm{~g}^{-1}$ soil day ${ }^{-1}$ respectively) compared $\mathrm{T}_{8}$ (weed free check) (4.05 $\mu \mathrm{g} \mathrm{NH}_{4} \mathrm{~N} \mathrm{~g}^{-1}$ soil day ${ }^{-1}$ ).

\section{Post emergence application}

At 7 DAT, T $_{8}$ (weed free check) was recorded significantly higher urease activity $(4.74 \mu \mathrm{g}$ $\mathrm{NH}_{4} \mathrm{~N} \mathrm{~g}^{-1}$ soil day ${ }^{-1}$ ) followed by $\mathrm{T}_{9}$ (weedy check) and $\mathrm{T}_{7}$ (Atrazine at $1 \mathrm{~kg} \mathrm{ha}^{-1}+2 \mathrm{IC}+1$ HW). All the herbicide treatments recorded significantly lower urease activity compared to $\mathrm{T}_{8}$ (weed free check). $\mathrm{T}_{6}$ (Atrazine at $1 \mathrm{~kg}$ $\mathrm{ha}^{-1} \mathrm{fb}$ Imazathapyr at $\left.75 \mathrm{~g} \mathrm{ha}^{-1}\right)$ was recorded the lowest urease activity $\left(2.73 \mu \mathrm{g} \mathrm{NH}_{4} \mathrm{~N} \mathrm{~g}^{-1}\right.$ soil day ${ }^{-1}$ ).

At $15 \mathrm{DAT}, \mathrm{T}_{5}$ (Atrazine at $1 \mathrm{~kg} \mathrm{ha}^{-1} \mathrm{fb}$ Halosulfuron at $90 \mathrm{~g} \mathrm{ha}^{-1}$ ) was recorded significantly higher urease activity $(4.63 \mu \mathrm{g}$ $\mathrm{NH}_{4} \mathrm{~N} \mathrm{~g}^{-1}$ soil day ${ }^{-1}$ ) which was on par with $\mathrm{T}_{8}$ (weed free check) (4.62 $\mu \mathrm{g} \mathrm{NH}_{4} \mathrm{~N} \mathrm{~g}^{-1}$ soil day $^{-1}$ ). Whereas, $\mathrm{T}_{1}$ (Atrazine at $1 \mathrm{~kg} \mathrm{ha}^{-1} \mathrm{fb}$ Glyphosate at $2.5 \mathrm{~kg} \mathrm{ha}^{-1}$ ) was recorded significantly lower urease activity (3.69 $\mu \mathrm{g}$ $\mathrm{NH}_{4} \mathrm{~N} \mathrm{~g}^{-1}$ soil day ${ }^{-1}$ ) compared to $\mathrm{T}_{8}$ (weed free check) which was on par with $\mathrm{T}_{2}$ and $\mathrm{T}_{6}$

At different growth stages of maize both after pre and post emergence application of herbicides, all the enzymatic activities in the soil was reduced as compared to untreated plots due to the toxic effect of herbicides molecule on the microbial population. The results were conformity with that of NurMasirah et al. (2013) and Sebiomo et al. (2011). But after some days the enzymatic activity as well as the microbial population increased due to commensalism and proto cooperation. Mariusz et al. (2013) also observed similar kind of results.

\section{References}

Casida, L.E., Klein, D.A. and Santoro, T. 1964. Soil dehydrogenase activity. Soil Sci., 98: 371-376.

Chougala, V.R. 2013. Evaluation of sequential application of pre and post emergence herbicides for weed management in maize, M.Sc. (Agri) Thesis, University of Agricultural Sciences Dharwad, Karnataka (India).

Donald, C.M. 1962. In search of yield. J. Australian Inst. Agric. Sci., 28: 171178.

Evazi, Z. and Tabatabai, M.A. 1979. Phosphatase in soil. Soil Biol. Biochem., 9: 167-172.

Ishrat, D.H., Hunshal, C.S., Malligwad, L.H. and Chimmad, V.P. 2012. Effect of pre and post emergence herbicides on weed control in maize. Karnataka J. Agri. Sci., 25(3): 392-394.

Kamble, T.C., Kakade, S.U., Nemade, S.U., Pawar, R.V. and Apotikar, V.A. 2005. Integrated weed management in hybrid maize. Crop Res., 29(3): 396-400.

Misra, A. and Tosh, G.C. 1979. Chemical weed control studies on dwarf wheat, $J$. Res., (Orissa Unv. of Agrl. and Tech.), 10: 1-6.

Pancholy, S.K. and Rice, E.L. 1973. Soil enzymes in relation to old field succession: amylase, invertase, cellulose, dehydrogenase and urease. Soil Sci. Soc. America, 37: 47-50.

Patil, P.I. 2014. Effect of sequential 
application of pre and post emergence herbicides in rainfed maize (Zea mays L). M. Sc. (Agri.) Thesis, University of Agricultural Sciences Dharwad, Karnataka (India).

Shantveerayya, H., Agasimani, C.A., Halikatti, S.I., Babu, R., Patil, C.R. and Ningnur, B.T. 2012. Effect of herbicide weed control and productivity of maize
(Zea mays L.). Karnataka J. Agri. Sci., 25(1): 137-139.

Singh, S., Hiremath, S.M., Yadav, S.L., Meena, L.K. and Chouhan, B.S. 2014. Growth and yield of maize as influenced by integrated weed management practices. Annals of Agri. Biol. Res., 19(3): 422-424.

\section{How to cite this article:}

Tapas Ranjan Sahoo, U.K. Hulihalli, R.K. Paikaray and Mohapatra, U. 2017. Microbial Response to Different Herbicides Application in Maize for Effective Weed Management. Int.J.Curr.Microbiol.App.Sci. 6(2): 735-743. doi: http://dx.doi.org/10.20546/ijcmas.2017.602.081 